\title{
INVESTIGATION OF MAGNETIC STRUCTURES AND PHASE TRANSITIONS IN $\mathrm{NiS}_{2}$ BY ${ }^{61} \mathrm{Ni}-\mathrm{MÖSSBAUER}$ SPECTROSCOPY
}

\author{
G. CZJZEK, J. FINK, H. SCHMIDT
}

Institut für Angewandte Kernphysik

Kernforschungszentrum Karlsruhe (GFR)

\author{
G. KRILL, F. GAUTIER, M. F. LAPIERRE and C. ROBERT \\ Laboratoire de Structure Electronique des Solides, \\ Université Louis-Pasteur, Strasbourg, France
}

\begin{abstract}
Résumé. - Les études des interactions hyperfines magnétiques dipolaires et électriques quadru-

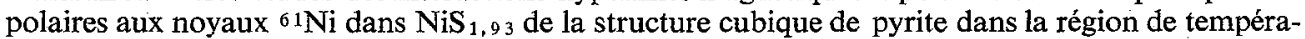
tures $4,2 \mathrm{~K} \leqslant T \leqslant 50 \mathrm{~K}$ ont mené aux résultats suivants. Entre $30,9 \mathrm{~K}$ et $T_{\mathrm{N}}=44,5 \mathrm{~K}$ les moments magnétiques des ions nickel sont alignés parallèles aux axes locaux de symétrie trigonale. Au-dessous de $30,9 \mathrm{~K}$ nous trouvons deux sites de nickel distingués par des angles différents entre la direction du moment magnétique et l'axe local de symétrie. Pour à peu près $50 \%$ des ions nickel la direction du moment commence à changer à $(30,9 \pm 0,1) \mathrm{K}$, pour les autres à $(30,2 \pm 0,1) \mathrm{K}$. D'une analyse des champs hyperfins magnétiques au sens des interactions dipôle-dipôle anisotropes et des rapports des constantes de couplage quadrupolaire nous dérivons des angles de $77^{\circ}$ et de $39^{\circ}$ entre les moments magnétiques et les axes de symétrie pour les deux sites. Qualitativement les mêmes résultats sont obtenus pour $\mathrm{NiS}_{1,96}$ et $\mathrm{NiS}_{2,00}$ dans la région de basses températures avec des petits changements de températures critiques et des paramètres d'interactions hyperfines. Il paraît, pourtant, que $T_{\mathrm{N}}$ croît rapidement avec la teneur de soufre. Une distribution statique des moments magnétiques ou des effets de relaxation mène à un élargissement significatif des raies d'absorption entre $50 \mathrm{~K}$ et $T_{\mathrm{N}}$.
\end{abstract}

Abstract. - Measurements of the magnetic dipole and electric quadrupole interactions at $61 \mathrm{Ni}$ in $\mathrm{NiS}_{1.93}$ with cubic pyrite structure in the temperature region $4.2 \mathrm{~K} \leqslant T \leqslant 50 \mathrm{~K}$ have yielded the following results. Between $30.9 \mathrm{~K}$ and $T_{\mathrm{N}}=44.5 \mathrm{~K}$ the magnetic moments on Ni ions are aligned parallel to the local trigonal symmetry axis. Below $30.9 \mathrm{~K}$ we find two nickel sites distinguished by different angles between the direction of the magnetic moment and the local symmetry axis. For about $50 \%$ of the nickel ions the moment direction begins to change at $(30.9 \pm 0.1) \mathrm{K}$, for the other ones at $(30.2 \pm 0.1) \mathrm{K}$. From an analysis of the magnetic hyperfine fields in terms of anisotropic dipole-dipole interactions and from the ratios of the effective quadrupole constants we derive values of $77^{\circ}$ and $39^{\circ}$ for the angles between magnetic moments and symmetry axes at the two sites. Qualitatively the same results are obtained for $\mathrm{NiS}_{1.96}$ and $\mathrm{NiS}_{2.00}$ in the low-temperature region with small changes of critical temperatures and hyperfine-interaction parameters. However, $T_{\mathrm{N}}$ appears to increase rapidly with increasing sulfur content. Either a static distribution of magnetic moments or relaxation effects lead to a significant broadening of the absorption lines in the temperature region between $50 \mathrm{~K}$ and $T_{\mathbb{N}}$.

Recent studies of $\mathrm{NiS}_{2}$ with cubic pyrite structure by neutron diffraction [1] and by measurements of magnetic susceptibility and electrical resistivity $[2,3]$ have established the occurrence of two magnetic phase transitions in this compound for compositions slightly deficient in sulfur content. Near the composition $\mathrm{NiS}_{1.9}$ antiferromagnetic order is found below $T_{\mathrm{N}} \sim 40-50 \mathrm{~K}$, and the material becomes weakly ferromagnetic below $T_{\mathrm{c}} \sim 30-31 \mathrm{~K}$. For both phases, the magnetic moment arrangements have not yet been determined.

We have now investigated the temperature dependence of hyperfine interactions at ${ }^{61} \mathrm{Ni}$ nuclei in a sample with composition $\mathrm{NiS}_{1.93}$ in the temperature range $4.2 \mathrm{~K} \leq T \leq 50 \mathrm{~K}$ by Mössbauer spectroscopy.
The experimental results are summarized in figure 1 . Some preliminary results have also been obtained with samples of $\mathrm{NiS}_{1.96}$ and $\mathrm{NiS}_{2.00}$, prepared by high-pressure synthesis with nickel enriched to $75 \%{ }^{61} \mathrm{Ni}$. X-ray diffraction and susceptibility measurements of all samples gave results in agreement with those reported previously [2,3]. The apparatus, experimental techniques, and data analysis procedures were the same as described in [4].

For $T \geqslant 45 \mathrm{~K}$ (paramagnetic phase) we find a slightly broadened single line. By comparison with the unsplit absorption spectrum of a NiV (14\%) absorber, taken with the same source immediately after the spectrum of $\mathrm{NiS}_{1.93}$, we deduce a quadrupole coupling constant $\left|v_{\mathrm{Q}}\right|=\left|e^{2} q Q / h\right|=(8 \pm 3) \mathrm{MHz}$. 


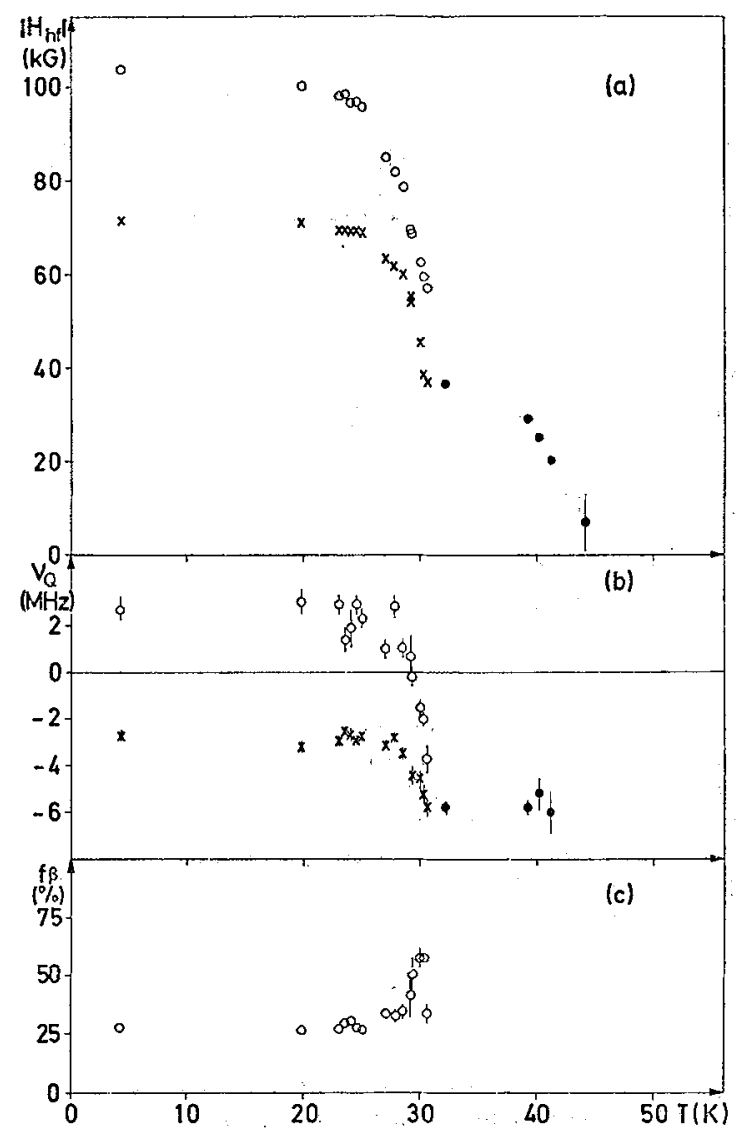

Fig. 1. - Temperature dependence of hyperfine interaction parameters in $\mathrm{NiS}_{1.93}$. antiferromagnetic phase, $x \alpha$-sites, $\bigcirc \beta$-sités. (a) Magnetic hyperfine field, $(b)$ effective quadrupole coupling constant, (c) fraction of ${ }^{61} \mathrm{Ni}$-nuclei occupying $\beta$-sites.

For $31 \mathrm{~K} \leq T \leq 44 \mathrm{~K}$ (antiferromagnetic phase) the ${ }^{61} \mathrm{Ni}$ nuclei experience a magnetic hyperfine field $H_{\mathrm{hf}}$ whose temperature dependence is quite well described by the molecular-field approximation with $T_{\mathrm{N}}=44.5 \mathrm{~K}$ and $\left|H_{\mathrm{hf}}(T=0)\right|=47 \mathrm{kG}$. The effective quadrupole coupling constant, $v_{\mathrm{Q}}^{\text {eff }}=\frac{1}{2}\left(3 \cos ^{2} \theta-1\right) \cdot e^{2} q Q / h$, where $\theta$ is the angle between the direction of $\mathbf{H}_{\mathrm{hf}}$ and the principal axis of the field-gradient tensor, is given by $v_{\mathrm{Q}}^{\text {eff }}=(-5.8 \pm 0.2) \mathrm{MHz}$, independent of temperature.

From the fact that we find the same magnetic hyperfine field and the same, value of $v_{Q}^{\text {eff }}$ for all ${ }^{61} \mathrm{Ni}$ nuclei in conjunction with the results of neutron diffraction [1] we conclude that the magnetic moment on any nickel ion is parallel to that (111)-direction which is the local trigonal symmetry axis. That is, $\theta=0$, and the experimental value of $v_{\mathrm{Q}}^{\text {eff }}$ is equal to $v_{\mathrm{Q}}=e^{2} q Q / h$.

At $(30.9 \pm 0.1) \mathrm{K}$ a discontinuity of the slope of the $H_{\mathrm{hr}}$-vs.-T-curve is observed for about $50 \%$ of the ${ }^{61} \mathrm{Ni}$ nuclei ( $\beta$-sites), whereas the field on the other nuclei $(\alpha$-sites) remains practically constant down to $(30.2 \pm 0.1) \mathrm{K}$. For temperatures below $30.2 \mathrm{~K}$ the field on all ${ }^{61} \mathrm{Ni}$ nuclei deviates from the curve extrapolated from the antiferromagnetic phase (Fig. 1a). However, in the entire temperature region below
$30.9 \mathrm{~K}$, we find two nickel sites with different values of $H_{\mathrm{hf}}$ (Fig. $1 a$ ) and of $v_{\mathrm{Q}}^{\text {eff }}$ (Fig. 1b). The effective quadrupole coupling constants change gradually between $30.9 \mathrm{~K}$ and $29 \mathrm{~K}$. Below $29 \mathrm{~K}$ we find the values $v_{\mathrm{Q}, \alpha}^{\text {eff }}=(-2.8 \pm 0.1) \mathrm{MHz}, v_{\mathrm{Q}, \beta}^{\text {eff }}=(2.4 \pm 0.2) \mathrm{MHz}$, independent of temperature. Between about $29 \mathrm{~K}$ and $25 \mathrm{~K}$ the fraction of ${ }^{61} \mathrm{Ni}$ nuclei in $\beta$-sites decreases gradually from about $50 \%$ to about $25 \%$ (Fig. $1 c$ ).

The changes of $H_{\mathrm{hf}}$ near $30 \mathrm{~K}$ could be caused by similar changes of the values of the nickel moments. However, this would lead to corresponding changes of the intensities of magnetic powder diffraction lines which have not been reported in the literature. Therefore it seems more likely that the observed effects are caused by rotations of the magnetic moments in conjunction with anisotropic hyperfine interactions.

The Hamiltonian for the magnetic hyperfine interaction in a coordinate system with the $z$-axis parallel to the direction of the magnetic moment can be written in the form :

$$
\begin{aligned}
H_{\text {magn }}= & \mu_{\text {nucl }}\left[A_{0}+\frac{1}{2} A_{\mathrm{D}} \cdot\left(3 \cos ^{2} \theta-1\right)\right] S_{z} \cdot I_{z}- \\
& -\frac{3}{4} \mu_{\text {nucl }} A_{\mathrm{D}} \cdot \sin \theta \cdot \cos \theta \cdot S_{z} \cdot\left(I_{+}+I_{-}\right)
\end{aligned}
$$

with the usual notation for the nuclear spin operators $I_{z}, I_{+}=I_{x}+\mathrm{i} I_{y}, I_{-}=I_{x}-\mathrm{i} I_{y}$ and the electronic spin operator $S_{z}$. The angle $\theta$ is again that between the moment direction and the symmetry axis. The quantity $A_{0}$ is determined by the isotropic part of the hyperfine interaction : the contact term and the interaction with the orbital moment. The anisotropic part, $A_{\mathrm{D}}$, originates from the dipole-dipole interaction between electronic and nuclear spins. The orbital term may also contribute to the anisotropy if the electronic g-tensor is anisotropic [5]. As EPR measurements on nickel ions in $\mathrm{Fe}_{1-x} \mathrm{Ni}_{x} \mathrm{~S}_{2}$ with the same structure have shown that in this case $\mathrm{g}_{x}$ is very nearly the same as $\mathrm{g}_{z}$ [6], we assume that the anisotropy in $\mathrm{NiS}_{2}$ can be ascribed entirely to the dipole-dipole interaction.

Treating the anisotropic part of the Hamiltonian, eq. (1), as a perturbation to second order, we can express the magnetic hyperfine interaction in terms of an effective magnetic hyperfine field, given by

$$
\begin{aligned}
H_{\mathrm{hf}}^{\text {eff }}= & -A_{0} \cdot\left\langle S_{z}>\cdot\left[1+\frac{1}{2}\left(3 \cos ^{2} \theta-1\right) \cdot\left(A_{\mathrm{D}} / A_{0}\right)\right.\right. \\
& \left.+(9 / 8) \cdot \cos ^{2} \theta \cdot\left(1-\cos ^{2} \theta\right) \cdot\left(A_{\mathrm{D}} / A_{0}\right)^{2}\right] \cdot
\end{aligned}
$$

As no significant structural changes have been observed in the critical temperature region, we analyze our results assuming changes only of the moment directions, that is of the angle $\theta$, not of the intrinsic hyperfine interaction parameters $A_{0}, A_{\mathrm{D}}$ and $v_{\mathrm{Q}}$. From a comparison of the experimental values of the hyperfine fields $H_{\mathrm{hf}, \alpha}^{\text {eff }}$ and $H_{\mathrm{hf}, \beta}^{\text {eff }}$ at $4.2 \mathrm{~K}$ with the value extrapolated from the antiferromagnetic phase, which we set equal to $H_{\mathrm{hf}}^{\text {eff }}(\theta=0)=-A_{0} \cdot\left\langle S_{z}\right\rangle \cdot\left[1+A_{\mathrm{D}} / A_{0}\right]$ and using the ratio

$v_{\mathrm{Q}, \alpha}^{\mathrm{eff}} / v_{\mathrm{Q}, \beta}^{\mathrm{eff}}=\left(3 \cdot \cos ^{2} \theta_{\alpha}-1\right) /\left(3 \cdot \cos ^{2} \theta_{\beta}-1\right)=-1.16$ 
we obtain the values $\theta_{\alpha}=39^{\circ}, \theta_{\beta}=77^{\circ}$, both with an uncertainty of about $10 \%$. The same values for these angles are obtained from a comparison of $v_{Q, \alpha}^{\text {eff }}$ and $v_{\mathbf{Q}, \beta}^{\text {eff }}$ with the value of $v_{\mathrm{Q}}$ measured in the antiferromagnetic phase. This agreement lends support to the assumptions involved in the derivation.

For the hyperfine interaction parameters we derive the values :

$\left|A_{0}<S_{z}>\right|=87 \mathrm{kG}, \mid A_{\mathrm{D}} \cdot\left\langle S_{z}>\right|=40 \mathrm{kG}$, with $A_{\mathrm{D}} / A_{0}<0$. The dipole-dipole term $A_{\mathrm{D}}$ is closely related to the contribution of the valence electrons to the electric-field gradient, both quantities being proportional to $\left\langle\left(3 \cos ^{2} \theta-1\right) / r^{3}\right\rangle$ [5]. Using $\left\langle S_{z}\right\rangle=1.2 \mu_{\mathrm{B}}$ as given by Hastings and Corliss [1], we derive $:\left|\left\langle\left(3 \cos ^{2} \theta-1\right) / r^{3}\right\rangle_{\text {magn }}\right|=3.6 \times 10^{24} \mathrm{~cm}^{-3}$.

For the quadrupole coupling constant we have to account for the screening factor $(1-R)$. As no calculation of the value of $R$ for $\mathrm{Ni}^{2+}$ is known to us, we estimate it from a comparison with iron to be about 0.3 . Then we obtain

$\left\langle\left(3 \cos ^{2} \theta-1\right) / r^{3}\right\rangle_{\text {quadrup }}=-1.5 \times 10^{24} \mathrm{~cm}^{-3}$.

The discrepancy between these two results may have several causes : $(a)$ an anisotropic contribution of the electronic orbital moment, $(b)$ a larger value of the nickel moment than that given by Hastings and Corliss, (c) a positive lattice contribution to the electric field gradient, partially compensating the gradient due to the "valence electrons, and $(d)$ a different radial distribution of spin and charge densities.

In the samples of composition $\mathrm{NiS}_{1.96}$ and $\mathrm{NiS}_{2.00}$ we find essentially the same phenomena as in $\mathrm{NiS}_{1.93}$ in the temperature region below $45 \mathrm{~K}$. With increasing sulfur content, the critical temperature for the moment rotation increases slightly to about $34 \mathrm{~K}$ for $\mathrm{NiS}_{2.00}$. The magnetic hyperfine fields at $4.2 \mathrm{~K}$ decrease only by a few percent. As the bulk magnetization decreases very rapidly with increasing sulfur content in this concentration region, the directions of the magnetic moments must change to an antiferromagnetic arrangement.

The Néel temperature increases rapidly with the sulfur content. In $\mathrm{NiS}_{2.00}$ we found so far a magnetic splitting up to $70 \mathrm{~K}$. At the same time, the absorption lines are broadened strongly for $T \geqslant 50 \mathrm{~K}$. This can be due either to a static distribution of magnetic moments or to relaxation effects.

\section{References}

[1] Hastings, J. M. and CoRliss, L. M., IBM J. Res. Develop. 14 (1970) 227.

[2] Gautier, F., Krill, G., Lapierre, M. F. and Robert, C., Solid State Commun. 11 (1972) 1201.

[3] Gautier, F., Krill, G., Lapierre, M. F. and Robert, C., J. Phys. C : Solid State Phys. 6 (1973) L 320.

[4] Fink, J., Czjzek, G., Schmidt, H., Ruebenbauer, K.,
COEY, J. M. D. and BRUSETTI, R., to be published in J. Physique.

[5] Johnson, C. E., in Hyperfine Structure and Nuclear Radiations, Matthias, E. and Shirley, D. A., eds. (NorthHolland Amsterdam, 1968) p. 226.

[6] Chandler, R. N. and Bené, R. W., Phys. Rev. B 8 (1973) 4979. 